\title{
Factors Contributing to Emotional Distress among Postpartum Mothers with Newborns at Newborn Unit Kenyatta National Hospital, Kenya
}

\author{
Omulo O. Wycliffe ${ }^{1}$, Cheptum Jebet Joyce ${ }^{2}$, Mageto G. Irene Mageto ${ }^{3}$ \\ ${ }^{I}$ BSc Nursing Student at the Stime of the Study, ${ }^{2}$ Lecturer, Midwifery and Obsteric Nursing, \\ ${ }^{3}$ Lecturer, Mental health and Psychiatric Nursing, School of Nursing Sciences, University of Nairobi, \\ P.O Box 19676-00202, Kenyatta National Hospital Nairobi, Kenya
}

\begin{abstract}
Background: The expectation of a woman during pregnancy is to have a healthy live bay with no complications. Admission of a newborn baby to the newborn unit is distressing to the parents, more so in cases where there is little or no support from the health care team and other players.

Objective: To establish factors contributing to emotional distress among postpartum mothers with newborns at newborn unit Kenyatta National Hospital, Kenya.

Method: This was a descriptive cross-sectional study employing a quantitative method by use of an interviewer-administered questionnaire. The study was conducted among 59 postpartum mothers with newborns at the Newborn Unit Kenyatta National Hospital. Simple random sampling technique was employed and data collected using a pretested semi-structured questionnaire. Data was analyzed using Statistical Package for The Social SPSS version 25 software. Qualitative data was coded, categorized into themes and thematic analysis done.
\end{abstract}

Results: The factors contributing to maternal emotional distress were low levels of education primary $14(23.7 \%)$ and secondary $23(39.0 \%)$ and unemployment $27(45.8 \%)$. In addition, lengthy NBU stays $34(57.6 \%)$, ineffective communication patterns $18(30.5 \%)$ and null communication $9(15.3 \%)$ between mothers and the healthcare givers contributed to emotional distress.

Conclusion: There are sociodemographic, socioeconomic, and hospital factors contributing to maternal emotional distress.

Keywords: Emotional distress, postpartum mothers, newborn unit, factors, baby.

\section{Introduction}

Postpartum period is the duration immediately after birth of a child and the expulsion of placenta extending

\section{Corresponding Author:}

Joyce Jebet

Lecturer, Midwifery and Obsteric Nursing, School of Nursing Sciences, University of Nairobi, P.O Box 19676-00202, Kenyatta National Hospital Nairobi,

Kenya

e-mail: jjcheptum@gmail.com

Tel: +254721475165 to about 6 weeks ${ }^{1}$. During this period, the maternal body undergoes a lot of physiological changes to return into the non-pregnant state. Mothers face a lot of challenges during the postpartum period ${ }^{2}$. These include need for social support, issues with breast feeding (especially first-time mothers) and need for help with postpartum emotional distress among others. Globally, emotional distress during the postpartum period will impact negatively on both the maternal and the newborn health and well-being ${ }^{3}$. Emotional distress is the second most common cause of maternal deaths in United States of America (USA) with about $20 \%$ of the affected population committing suicide each year ${ }^{4}$. Maternal 
emotional distress negatively impacted on the motherchild bonding leading to impaired neurodevelopment among the children in China ${ }^{5}$. It was reported that emotionally distressed postpartum mothers have higher chances of early breastfeeding termination. $40 \%$ of children in such cases were ending up underweight and stunted in growth. Apart from increasing mortality rate with emotional distress, mothers also showed less affectionate manners towards their newborns in $\mathrm{USA}^{6}$. In Kenya, the prevalence of infanticides among emotionally distressed mothers as at $19 \%$ and 1 out of 10 emotionally distressed mothers is likely to commit suicide. $^{7,8}$

Newborn unit (NBU) admissions have been associated with elevated emotional distress among the postpartum mothers ${ }^{6}$. It is believed that the NBU environment, the prolonged duration of hospital stays due to newborn admission among other factors contribute to heightened emotional distress among the mothers.

Sociodemographic and socioeconomic factors have been found to contribute to emotional distress among postpartum mothers ${ }^{9}$. These include being household heads, low level of education and financial constraints ${ }^{10}$. Hispanic women living in the rural areas of Southern California were more likely to develop emotional distress during the postpartum period due to low level of education, unemployment, caesarean mode of delivery and being a primipara ${ }^{11}$. Mothers who experienced suicidal thoughts during pregnancy, those who were exposed to domestic violence, those who had unplanned pregnancies and a history of a previous psychiatric illness were at a higher risk of developing emotional distress ${ }^{12}$.

A few studies have been conducted to establish the factors which may lead to the heightened emotional distress among the NBU mothers. These are different from the causes of newborn admissions themselves. World Health Organization reports poverty, low social support, exposure to violence and extreme stress among other factors as the causes of emotional distress among such mothers ${ }^{13}$. Several hospital factors have been found to contribute to emotional distress among postpartum mothers with babies at the NBU with regards to the complexity of such environments ${ }^{14}$. Concerns that the health care team may misunderstand the newborn's needs and mothers feeling of lack of information on the diagnosis or treatment of their newborns contributed to maternal distress ${ }^{15}$. In a systemic review of parental needs for mothers with NICU newborns in US, there was lack of communication of information, maternal involvement in the newborn's care and lack of positive perception by and interaction with the NICU staff ${ }^{16}$. In a descriptive study in Turkey (state hospital Canakkale city), information communication, empathic emotional support and mothers' involvement in the treatment and diagnosis of their newborns lacked among the health care team ${ }^{17}$.

Lack of communication from health care staff was yet still a stressor in the NBU among the mothers of Midwestern ${ }^{6}$. Mothers felt alienated from care of their newborns. Poor bedside manner of the medical staff was also of major concern. Half of the mothers were being informed of the newborn's disease in inappropriate situations and most of them complained of negative attitude among health care professionals ${ }^{18}$. The unfavorable hospital conditions, not being able to see their newborns whenever they wished and not being able to get enough information regarding their newborns were also of major concerns.

With regard to Kenya's Sustainable Development Goal (SDG) 3 of ensuring healthy lives and promoting well-being for all at all ages, maternal and hence neonatal health and well-being is vital and relying on the country's efforts in the delivery of health care services to her citizens. Kenyatta National Hospital (KNH) therefore being the largest referral health facility receiving both direct admissions and referred cases from all across the country, it was very important to identify those postpartum mothers at potential risk for emotional distress and appropriate interventions implemented. In ensuring the same, the findings from this study may help establish those factors contributing to emotional distress and help put up the strategies to reduce such incidences.

\section{Materials and Method}

This was a descriptive cross-sectional study employing a quantitative method to establish factors contributing to emotional distress among postpartum mothers with babies at the newborn unit at Kenyatta National Hospital. A sample size of 59 mothers who met the inclusion criteria were randomly selected to participate in the study. Data was collected using an interviewer administered questionnaire. Data was cleaned, coded and entered in SPSS version 25 and analyzed. Descriptive statistics was reported and data was presented by use of figures and tables. Ethical approval 
was sought from Kenyatta National Hospital-University of Nairobi (KHN-UON) Ethics and Research Committee and permission at the Kenyatta hospital granted by the head of department obstetrics and gynecology.

\section{Results}

On sociodemographic factors, majority of the respondents 17(28.8\%) were aged between 18-23 years old. The mothers who were married were $32(54.2 \%)$. According to the findings, secondary $23(39.0 \%)$ and primary $14(23.7 \%)$ respectively were the levels of education attained by most mothers. The mothers residing far and very far from the hospital were $34(57.6 \%)$ and $17(28.8 \%)$ respectively. Those who had fairly good experience (uneventful) during pregnancy were $34(57.6 \%)$ while those with challenging and extremely stressful experiences were $17(28.8 \%)$ and $8(13.6 \%)$ respectively. The primiparous mothers were $23(39 \%)$ while $36(61 \%)$ were multiparous. Those who delivered through caesarian section and spontaneous vertex delivery were $25(42.4 \%)$ and 34(57.6\%) respectively.

On socioeconomic factors,majority $27(45.8 \%)$ of the mothers were unemployed. Most mothers 34(57.6\%) had income levels below 10,000 shillings. A larger percentage $39(66.1 \%)$ of the mothers reported to be the sole breadwinners in their families. On theother hand, mothers reported their past medical history and majority $37(45.1 \%)$ of the mothers had not previously suffered from any psychiatric illnesses.

On hospital factors, 34(57.6\%) of the mothers had babies admitted to NBU for durations between 4-6 days while those above 6 days were at 25(42.4\%). According to the findings, majority of the mothers $33(55.9 \%)$ and $20(33.9 \%)$ found the explanations regarding the baby diagnoses as helpful and confusing respectively. Most mothers 31(52.5\%) and 17(28.8\%) were worried and confused respectively upon first incubator sight of the baby.For15(25.4\%) of the mothers, theNBU policies were hindering while $20(33.9 \%)$ found them as stressful with only $24(40.7 \%)$ reporting the policies as protective. Majority of the mothers $28(47.5 \%)$ and $15(25.4 \%)$ received updates on baby's progress upon inquiry and weekly respectively. The findings showed $18(30.5 \%)$ and $9(15.3 \%)$ of the mothers reporting the mothers-caregivers' communications as ineffective and null respectively. Mothers who found the hospitals counseling services as insufficient were 29(49.2\%), $22(37.3 \%)$ as beneficial while $8(13.6 \%)$ did not receive any form of counselling. All the findings are as shown in the table 1 below.

Table 1: Sociodemographic factors contributing to emotional distress among the respondents

\begin{tabular}{|c|c|c|}
\hline Characteristics & $\begin{array}{c}\text { Frequency } \\
(n=59)\end{array}$ & $\begin{array}{c}\text { Percentage } \\
(\%)\end{array}$ \\
\hline \multicolumn{3}{|l|}{ Sociodemographic Factors } \\
\hline \multicolumn{3}{|l|}{ Age } \\
\hline $18-23$ & 17 & 28.8 \\
\hline $24-29$ & 15 & 25.4 \\
\hline $30-35$ & 16 & 27.2 \\
\hline $36-41$ & 11 & 18.6 \\
\hline \multicolumn{3}{|l|}{ Education } \\
\hline Primary & 14 & 23.7 \\
\hline Secondary & 23 & 39.0 \\
\hline College & 13 & 22.0 \\
\hline University & 5 & 8.5 \\
\hline Others & 4 & 6.8 \\
\hline \multicolumn{3}{|l|}{ Marital Status } \\
\hline Married & 35 & 54.2 \\
\hline Separated & 6 & 10.2 \\
\hline Single & 21 & 35.6 \\
\hline \multicolumn{3}{|l|}{ Residence } \\
\hline Far & 34 & 57.6 \\
\hline Very far & 17 & 28.8 \\
\hline Near & 8 & 13.6 \\
\hline \multicolumn{3}{|c|}{ Pregnancy Experience (Uneventful) } \\
\hline Good & 34 & 57.6 \\
\hline Challenging & 17 & 28.8 \\
\hline Extremely stressful & 8 & 13.6 \\
\hline \multicolumn{3}{|l|}{ Parity } \\
\hline Primiparous & 23 & 39.0 \\
\hline Multiparous & 36 & 61.0 \\
\hline \multicolumn{3}{|l|}{ Mode of delivery } \\
\hline Caesarian section & 25 & 42.4 \\
\hline Spontaneous vertex delivery & 34 & 57.6 \\
\hline \multicolumn{3}{|l|}{ Socioeconomic } \\
\hline \multicolumn{3}{|l|}{ Employment status } \\
\hline Employed & 11 & 18.6 \\
\hline Self-employed & 21 & 35.6 \\
\hline Unemployed & 27 & 45.8 \\
\hline \multicolumn{3}{|l|}{ Average Monthly Earnings } \\
\hline$<10,000$ & 34 & 57.6 \\
\hline $10,000-50,000$ & 24 & 40.7 \\
\hline$>50,000$ & 1 & 1.7 \\
\hline
\end{tabular}


Table 2: Hospital factors contributing to emotional distress among the respondents

\begin{tabular}{|c|c|c|}
\hline Characteristics & $\begin{array}{l}\text { Frequency } \\
\quad(n=59)\end{array}$ & $\begin{array}{c}\text { Percentage } \\
(\%)\end{array}$ \\
\hline \multicolumn{3}{|l|}{ Hospital Factors } \\
\hline \multicolumn{3}{|c|}{ Admission duration } \\
\hline 4-6 days & 34 & 57.6 \\
\hline$>6$ days & 25 & 42.4 \\
\hline \multicolumn{3}{|c|}{ Diagnoses explanations } \\
\hline Helpful & 33 & 55.9 \\
\hline Confusing & 20 & 33.9 \\
\hline Null & 5 & 8.5 \\
\hline \multicolumn{3}{|c|}{ Reaction to first incubator sight } \\
\hline Worried & 31 & 52.5 \\
\hline Confused & 17 & 28.8 \\
\hline Hopeful & 11 & 18.6 \\
\hline \multicolumn{3}{|l|}{ NBU policies } \\
\hline Hindering & 15 & 25.4 \\
\hline Stressful & 20 & 33.9 \\
\hline Protective & 24 & 40.7 \\
\hline \multicolumn{3}{|l|}{ Progress updates } \\
\hline Daily & 9 & 15.3 \\
\hline Weekly & 15 & 25.4 \\
\hline Upon inquiry & 6 & 10.2 \\
\hline No updates & 28 & 47.5 \\
\hline Others & 1 & 1.7 \\
\hline \multicolumn{3}{|c|}{ Mother-caregiver communication patterns } \\
\hline Effective & 28 & 47.5 \\
\hline Ineffective & 18 & 30.5 \\
\hline Null & 9 & 15.3 \\
\hline Others & 4 & 6.8 \\
\hline \multicolumn{3}{|c|}{ Hospital counselling services } \\
\hline Insufficient & 29 & 49.2 \\
\hline Beneficial & 22 & 37.3 \\
\hline Null & 8 & 13.6 \\
\hline
\end{tabular}

\section{Discussion}

Sociodemographic Factors: The finding of this study showed that majority of the mothers had attained primary and secondary levels of educations. Even though majority of the mothers found explanations on baby diagnosis by the health care givers as helpful, a good percentage perceived them as confusing with resultant anxiety. This can be attributed to the low level of education among the mothers with the characteristics of the health care givers a side. Lack of basic education can inhibit the understanding of even simplified information because even then, language barrier is a problem. This finding is in agreement with the study done by Kim and Dee where low levels of education contributed to maternal emotional distress ${ }^{19}$.

Socioeconomic Factors: The findings of this study showed that majority of the mothers were the sole breadwinners in their families (most of them were of small sizes). Most mothers were unemployed with a monthly income below 10,000 shillings. Due to the resultant financial strain in terms hospital costs and other incurred logistics, the additional stress is a contributory factor to maternal emotional distress. This can be attributed to non-engagement of the families in income generating activities and the unfavorable prevailing economic trends. This is in agreement with the previous study that being the head of the family was an additional stressor and a contributory factor to maternal emotional distress ${ }^{9}$. Other studies also found unemployment as a contributory factor to maternal emotional distress due to financial strains ${ }^{19}$.

Hospital Factors: In this study, majority of the mothers had babies staying in the NBU for durations between 4-6 days and a few exceeding 6 days. Such long stays were attributed to negative perceptions among the mothers with increased worries putting them at risk for maternal emotional distress. This is in agreement with the study that lengthy NBU stays increased strain on the mothers and their relationship with their babies increasing their chances of emotional distress ${ }^{20}$. This can be attributed to care givers lack of care optimization perhaps due to inadequate medical and human resources leading to lengthy NBU stays.

The finding s of this study showed a good number of the mothers finding the care givers' explanations regarding baby diagnosis confusing even though majority found them helpful. This can be attributed to the care givers characteristics such as use of technical medical language exacerbating their stress. This is in agreement with the finding $\mathrm{s}$ of the study that mothers' lack of information on diagnosis of their babies contributed to maternal emotional distress ${ }^{15}$. In another study, one out of five mothers had no idea on the diagnosis of their babies hence becoming emotionally distressed ${ }^{18}$. 
The new born unit (NBU) is unfamiliar setting where mothers feel lost and frustrated with the negative perceptions contributing to emotional distress ${ }^{20}$. The findings of this this study showed majority of the mothers feeling worried and confused on the first sight of their babies in the incubators. Most mothers also found the NBU policies stressful and hindering as far as taking part in care of their babies is concerned. This is in agreement with the findings of the study that NBU policies had set up impediments to the mothers for developing interactional skills with their babies hence exacerbating their emotional distress ${ }^{15}$. In some other study, NBU was found to be a complex environment raising anxiety and stress of the mothers. This can be due to lack of the health care givers' commitment in bringing to mothers an envisage of what NBU is in prior. Attributions can also be made to alienating NBU policies and regulations.

Majority of mothers were receiving updates on treatment progress of their babies weekly and only upon inquiry. This could be attributed to the care givers' lack of involvement of the mothers in the care of their babies and with the accompanying negative perceptions contributing to maternal emotional distress. This finding is in agreement with the study that there was lack of information to the mothers on treatment progress of the newborns in NBU leading to increased maternal emotional distress ${ }^{6,20}$.

In this study, majority of the mothers reported day to day communication between them and the healthcare givers as ineffective. This can be attributed to lack of involvement of the mothers by the healthcare givers in decision making and care of their babies and hence exacerbating their emotional distress. A tangible section of the mothers also reported the bed side manner of the healthcare providers as poor leading to stress among the mothers. These findings are in agreement with the study which found that poor bed side manner of the care givers, and lack of proper information communication gave the mothers a sense of alienation making them emotionally distressed $^{6}$. In another study, lack of information communication, lack of maternal involvement and lack of positive interaction between the mother and the care giver was contributory to emotional distress. ${ }^{4,20}$

The findings of this study showed that the counselling services provided by the hospital were insufficient according to majority of the mothers. A few reported the nonexistence of such services. This can be attributed to such counselling services not meeting the individualized needs of the mothers hence not meeting the threshold to aid towards emotional distress. This in agreement with the findings of the study that lack of emotional support by the healthcare team to help the mothers in expressing their feelings in coping with the babies' critical conditions was contributory to emotional distress ${ }^{3,17}$

\section{Conclusion}

In conclusion, sociodemographic factors such as low levels of education and being the head of the family as a mother were contributing to maternal emotional distress. Socioeconomic factors such as unemployment and low levels of income were also contributing to maternal emotional distress with baby receiving care at the NBU. Hospital factors contributing to maternal emotional distress included: - lengthy NBU stays, inconsistent updates on baby's treatment progress and poor explanations by the health care givers, stressful and hindering NBU policies and regulations, ineffective communication patterns between the mother and the care givers and frail counselling services offered by the hospital.

Conflict of Interests: We declare no conflict of interest.

Acknowledgement: We acknowledge all the personnel in the Obstetrics and Gynecology department of Kenyatta National Hospital and the mothers who participated in this study.

Source of Funding: This research project was selffunded.

Ethical Clearance: Ethical approval was obtained from the Kenyatta National Hospital-University of Nairobi Ethics and research Committee.

\section{References}

1. WHO, UNFPA, UNICEF. Pregnancy, Childbirth, Postpartum and New Born Care: A Guilde for Essential Practice. IWorld Heal Organ IIUNFPA IIIUNICEF IVWorld Bank ISBN [Internet]. 2015;978(2):92-4. Available from: www.who.int/ about/licensing/copyright_form/en/index.html

2. Lilo EA, Shaw RJ, Corcoran J, Storfer-Isser A, Horwitz SM. Does she think she's supported? Maternal perceptions of their experiences in the neonatal intensive care unit. Patient Exp J. 2016;3(1):15-24. 
3. Alkozei A, McMahon E, Lahav A. Stress levels and depressive symptoms in NICU mothers in the early postpartum period. J Matern Neonatal Med. 2014;27(17).

4. Binder W, L. Z, C. S. The father in the hallway: Differing parental roles and their relation to posttraumatic stress reactions in parents of NICU babies. Vol. 35, Neuropsychopharmacology. 2010.

5. González G, Moraes M, Sosa C, Umpierrez E, Duarte M, Cal J, et al. Maternal postnatal depression and its impact on child neurodevelopment: A cohort study. Rev Chil Pediatr. 2017;88(3).

6. Williams KG, Patel KT, Stausmire JM, Bridges C, Mathis MW, Barkin JL. The neonatal intensive care unit: Environmental stressors and supports. Int J Environ Res Public Health. 2018;15(1).

7. Ongeri L, Wanga V, Otieno P, Mbui J, Juma E, Stoep A Vander, et al. Demographic, psychosocial and clinical factors associated with postpartum depression in Kenyan women. BMC Psychiatry. 2018;18(1).

8. Mutua JN. Prevalence of Post-partum depression and anxiety among Mothers of preterm infants receiving Intensive Care in Newborn unit at Kenyatta National Hospital. 2017;

9. Alvarado Socarrás JL, Gamboa-Delgado EM, Trujillo Cáceres S, Rodríguez Forero S. Depressive symptoms and associated factors in caregivers of newborn infants hospitalized in a neonatal intensive care unit. Arch Argent Pediatr. 2017;115(2).

10. Tahirkheli NN, Cherry AS, Tackett AP, McCaffree MA, Gillaspy SR. Postpartum depression on the neonatal intensive care unit: Current perspectives. Vol. 6, International Journal of Women's Health. 2014.

11. Kim AR, Tak YR, Shin YS, Yun EH, Park HK, Lee HJ. Mothers' Perceptions of Quality of FamilyCentered Care and Environmental Stressors in Neonatal Intensive Care Units: Predictors of and Relationships with Psycho-emotional Outcomes and Postpartum Attachment. Matern Child Health J. 2020;24(5).

12. Narayan R. A study of the pattern of admissions and outcome in a neonatal intensive care unit at high altitude. Sri Lanka Journalof Child Heal. 2012;41(2).

13. WHO. WHO $\mid$ Neonatal mortality [Internet]. Who. 2017. Available from: http://www.who.int/gho/ child_health/mortality/neonatal_text/en/

14. Enke C, Oliva y Hausmann A, Miedaner F, Roth B, Woopen C. Communicating with parents in neonatal intensive care units: The impact on parental stress. Patient Educ Couns. 2017;100(4).

15. Baía I, Amorim M, Silva S, Kelly-Irving M, de Freitas C, Alves E. Parenting very preterm infants and stress in Neonatal Intensive Care Units. Early Hum Dev. 2016;101.

16. Turner M, Chur-Hansen A, Winefield H, Stanners M. The assessment of parental stress and support in the neonatal intensive care unit using the Parent Stress Scale - Neonatal Intensive Care Unit. Women and Birth. 2015;28(3).

17. Yilmaz F, Atay S, Arikan D, Güler ST. Evaluation of the effect of sociodemographic characteristics on the satisfaction of mothers in neonatal intensive care units in Turkey. Iran J Neonatol. 2016;7(1).

18. Yaman S, Altay N. Posttraumatic stress and experiences of parents with a newborn in the neonatal intensive care unit. Vol. 33, Journal of Reproductive and Infant Psychology. 2015. p. 140 52.

19. Kim Y, Dee V. Sociodemographic and Obstetric Factors Related to Symptoms of Postpartum Depression in Hispanic Women in Rural California. JOGNN - J Obstet Gynecol Neonatal Nurs. 2018;47(1).

20. Obeidat HM, Bond EA, Callister LC. The Parental Experience of Having an Infant in the Newborn Intensive Care Unit. J Perinat Educ. 2009;18(3). 